\title{
ORTHOGONALITY AND BOUNDARY INTERPOLATION
}

Adhemar Bultheel

Department of Computer Science

K.U. Leuven

Belgium

Pablo Gonzalez-Vera

Department of Mathematical Analysis

University of La Laguna

Tenerife, Spain

Erik Hendriksen

Department of Mathematics

University of Amsterdam

The Netherlands

Olav Njåstad

Department of Mathematical Sciences

University of Trondheim - NTH

Norway

\begin{abstract}
Let $\left\{\alpha_{n}: n=1,2, \ldots\right\}$ be a sequence of (not necessarily distinct) points on the unit circle. Set $\omega_{0}(z) \equiv 1, \omega_{n}(z)=\left(z-\alpha_{1}\right)\left(z-\alpha_{2}\right) \ldots\left(z-\alpha_{n}\right)$ for $n=1,2, \ldots$, and let $\mathcal{L}$ denote the linear space spanned by the functions $\left\{1 / \omega_{n}: n=0,1,2, \ldots\right\}$. Let $M$ be a quasi-definite linear functional on $\mathcal{L} \cdot \mathcal{L}$, and define the inner product $<,>$ by $<f, g\rangle=M\left(f g_{*}\right)$, where $g_{*}(z)=\overline{g(1 / \bar{z})}$. (In particular $M$ may be a positive definite functional given by $M(f)=\int_{-\pi}^{\pi} f\left(e^{i t}\right) d \mu(t)$, where $\mu$ is a measure such that all functions in $\mathcal{L} \cdot \mathcal{L}$ are $\mu$ integrable). Let $\left\{\varphi_{n}\right\}$ be an orthogonal system obtained form the base $\left\{1 / \omega_{n}\right\}$, and define the associated functions $\left\{\psi_{n}\right\}$ by $\psi_{n}(z)=M\left(\frac{t+z}{t-z}\left[\varphi_{n}(t)-\varphi_{n}(z)\right]\right)$. Interpolation of $\psi_{n} / \varphi_{n}$ to $M\left(\frac{t+z}{t-z}\right)$ (or to the formal series $\mu_{0} \neq 2 \sum_{m=0}^{\infty} \mu_{m} z \omega_{m-1}(z), \mu_{m}=M\left(1 / \omega_{n}\right)$ ) at interpolation points $\alpha_{k}$ is discussed.




\section{Introduction}

Let $\left\{\alpha_{n}\right\}$ be a sequence of (not necessarily distinct) points on the unit circle $\boldsymbol{T}=\{z \in$ $C:|z|=1\}$. We define the polynomials $\omega_{n}$ by

$$
\omega_{0}=1, \quad \omega_{n}(z)=\prod_{m=1}^{n}\left(z-\alpha_{m}\right), \quad n=1,2, \ldots .
$$

The linear space spanned by the functions $\left\{\frac{1}{\omega_{0}}, \frac{1}{\omega_{1}}, \ldots, \frac{1}{\omega_{n}}\right\}$ shall be denoted by $\mathcal{L}_{n}$, i.e.

$$
\mathcal{L}_{n}=\operatorname{Span}\left\{\frac{1}{\omega_{0}}, \frac{1}{\omega_{1}}, \ldots, \frac{1}{\omega_{n}}\right\}
$$

Further we define

$$
\mathcal{L}=\cup_{n=0}^{\infty} \mathcal{L}_{n}
$$

The functions in $\mathcal{L}_{n}$ are exactly the function that may be written in the form

$$
R(z)=\frac{p(z)}{\omega_{n}(z)}, p \in \Pi_{n}
$$

where $\Pi_{n}$ denotes the space of polynomials of degree at most $n$. (This follows from the basic facts about partial fraction decomposition.)

Let $M$ be a given linear functional defined at least on $\mathcal{L} \cdot \mathcal{L}$. (Note that $M$ is then defined on $\mathcal{L}$, since $\mathcal{L}=\mathcal{L} \cdot 1 \subset \mathcal{L} \cdot \mathcal{L}$.) The functional $M$ may in particular be defined by

$$
M[R]=\int_{-\pi}^{\pi} R\left(e^{i \theta}\right) d \mu(\theta),
$$

where $\mu$ is a finite Borel measure on $[-\pi, \pi]$ with the property that all the integrals $\int_{-\pi}^{\pi}\left[\omega_{m}\left(e^{i \theta}\right) \omega_{n}\left(e^{i \theta}\right)\right]^{-1} d \mu(\theta), m, n=0,1,2, \ldots$, exist.

For every function $f$ on $\hat{C}$ we define its substar transform $f_{*}$ by

$$
f_{*}(z)=\overline{f(1 / \bar{z})}
$$

We note that if $R \in \mathcal{L}_{n}$, then also $R_{*} \in \mathcal{L}_{n}$, since

$$
\left[\frac{1}{\omega_{m}(z)}\right]_{*}=(-1)^{m} \alpha_{1} \ldots \ldots \alpha_{m} \frac{z^{m}}{\omega_{m}(z)} .
$$


We define the Hermitian form $<,>$ on $\mathcal{L} \times \mathcal{L}$ by

$$
<P, Q>=M\left[P \cdot Q_{*}\right]
$$

We shall assume that this form is quasi-definite, i.e.

$$
<P, P>\neq 0, \quad \text { for } \quad P \in \mathcal{L}, P \not \equiv 0 \text {. }
$$

This is in particular the case for the form associated by (1.5), which is positive definite.

Let $\left\{\varphi_{n}\right\}$ be the (essentially unique) orthonormal system associated with the sequence $\left\{\frac{1}{\omega_{n}}\right\}$. We may write

$$
\varphi_{n}(z)=\frac{p_{n}(z)}{\omega_{n}(z)}, p_{n} \in \Pi_{n} .
$$

The functions of the second kind $\left\{\psi_{n}\right\}$ (associated with $\left\{\varphi_{n}\right\}$ ) are defined by

$$
\psi_{0}=1, \quad \psi_{n}(z)=M\left[\frac{t+z}{t-z}\left[\varphi_{n}(t)-\varphi_{n}(z)\right]\right], \quad n=1,2, \ldots .
$$

It is easily seen that the expression in the brackets [] belongs to $\mathcal{L}_{n}$ both as a function of $z$ and as a function of $t$, and $M$ is here meant to operate on its argument considered as a function of $t$. The function $\psi_{n}$ then belongs to $\mathcal{L}_{n}$, and it may be written as

$$
\psi_{n}(z)=\frac{q_{n}(z)}{\omega_{n}(z)}, q_{n} \in \Pi_{n} .
$$

Let us now assume that the functional $M$ is defined also for the functions $\eta_{z}^{(k)}(t)=\frac{z^{j} t^{k}}{t-z}$ for $j, k=0,1, z \in \hat{\boldsymbol{C}}-\boldsymbol{T}$. (By $M$ being defined for the function $f(z, t)$ for $z=\infty$ we mean that $M$ is defined for the function $f\left(\zeta^{-1}, t\right)$ for $\left.\zeta=0\right)$. Then $M$ is defined on the extended space $\tilde{\mathcal{L}} \cdot \tilde{\mathcal{L}}$, where

$$
\tilde{\mathcal{L}}=\operatorname{Span}\left\{\frac{1}{\omega_{n}(t)}, \frac{z^{j} t^{k}}{t-z}: \quad n=0,2,3, \ldots, j, k=0,1, \quad z \in \hat{C}-\boldsymbol{T}\right\} .
$$

We note that $M$ is then in particular defined for $t$ and $t^{2}$.

We may then define the Riesz-Herglotz transform $\Omega_{M}$ of $M$ by

$$
\Omega_{M}(z)=M\left[\frac{t+z}{t-z}\right] .
$$


(Here again $M$ operates on its argument considered as a function of $t$.) The functional $M$ defined by (1.5) is defined on $\tilde{\mathcal{L}} \cdot \tilde{\mathcal{L}}$. For the transform $\Omega_{M}$ of this functional we shall also write $\Omega_{\mu}$, thus

$$
\Omega_{\mu}(z)=\int_{-\pi}^{\pi} \frac{e^{i \theta}+z}{e^{i \theta}-z} d \mu(\theta) .
$$

The function $g_{t}(z)=\frac{t+z}{t-z}$ has formal Newton series expansions

$$
\frac{t+z}{t-z} \sim 1+2 \sum_{k=1}^{\infty} \frac{z \omega_{k-1}(z)}{\omega_{k}(t)}
$$

and

$$
\frac{t+z}{t-z} \sim-1+2 \sum_{k=1}^{\infty} \frac{\alpha_{k} t^{k} \omega_{k-1}(z)}{z^{k} \omega_{k}(t)} .
$$

(Cf. Section 2. For Newton series expansions, see also [12].) By operating formally by $M$ on (1.16) and (1.17) we get the Newton series expansions

$$
\Omega_{M}(z) \sim \mu_{0}+2 \sum_{k=1}^{\infty} \mu_{k} z \omega_{k-1}(z)
$$

and

$$
\Omega_{M}(z) \sim \nu_{0}+2 \sum_{k=1}^{\infty} \nu_{k} \frac{\omega_{k-1}(z)}{z^{k}},
$$

where the moments $\left\{\mu_{n}\right\},\left\{\nu_{n}\right\}$ are defined by

$$
\mu_{n}=M\left[\frac{1}{\omega_{n}(t)}\right], \quad n=0,1,2 \ldots
$$

and

$$
\nu_{0}=-\mu_{0}, \quad \nu_{n}=\alpha_{n} M\left[\frac{t^{n}}{\omega_{n}(t)}\right], \quad n=1,2, \ldots .
$$

We define the polynomials $P_{n}$ and $Q_{n}$ by

$$
P_{n}(z)=\mu_{0}+2 \sum_{k=1}^{n} \mu_{k} z \omega_{k-1}(z),
$$




$$
Q_{n}(z)=\nu_{0}+2 \sum_{k=1}^{n} \nu_{k} \frac{\omega_{k-1}(z)}{z^{k}} .
$$

For basic information concerning orthogonal functions associated with spaces $\mathcal{L}_{n}$ as defined above, see [8], cf. also [7,9].

We shall in this paper discuss some interpolation properties connecting the rational functions $\left\{\frac{\psi_{n}(z)}{\varphi_{n}(z)}\right\}$, the Riesz-Herglotz transform $\Omega_{M}(z)$ and the Newton series (1.18)-(1.19). It will be pointed out that $\left\{\frac{\psi_{n}(z)}{\varphi_{n}(z)}\right\}$ is an $(n, n)$ multipoint Padé approximant for $\Omega_{M}(z)$ at the interpolation table $\left\{0, \infty, \alpha_{1}, \alpha_{1}, \alpha_{2}, \alpha_{2}, \ldots, \alpha_{n-1}, \alpha_{n-1}, \alpha_{n}\right\}$. - Related connections where all the interpolation points are in the interior of the unit circle are discussed e.g. in $[4,5,6,7,16]$ and in references found there.

A special case of the situation treated in this paper is when the sequence $\left\{\alpha_{n}\right\}$ consists of a finite number of points cyclically repeated. The closely related (through the Cayley transform) cyclic situation when the points $\left\{\alpha_{n}\right\}$ lie on the real axis has been investigated e.g. in $[14,15,16]$ and in the references found there. When in particular two points are cyclically repeated (for convenience $\infty$ and 0 ), the orthogonal functions are orthogonal Laurent polynomials. (See e.g. [13,17]. Cf. also [3].) When all the points $\left\{\alpha_{n}\right\}$ coalesce to one point (for convenience $\infty$ ), the orthogonal functions are orthogonal polynomials. (See e.g. $[1,2,10]$.)

\section{Interpolation results}

In addition to the given interpolation points $\left\{\alpha_{n}\right\}$ on $T$, the point at the origin and at infinity will play a role in our discussion. We shall use the notation

$$
\alpha_{0}=0, \alpha_{\infty}=\infty
$$

Let $A_{n}$ denote the interpolation table $\left\{\alpha_{0}, \alpha_{1}, \ldots, \alpha_{n}\right\}$, and $\tilde{A}_{n}$ the table $\left\{\alpha_{\infty}, \alpha_{1}, \ldots, \alpha_{n}\right\}$. We note that the space $\mathcal{L}_{n}$ is independent of the order of the points $\left\{\alpha_{1}, \ldots, \alpha_{n}\right\}$ and the space $\mathcal{L}$ is independent of the order of the points $\left\{\alpha_{n}: n=1,2, \ldots\right\}$. We also note that for each $n$ the functions $\left\{\varphi_{0}, \varphi_{1}, \ldots, \varphi_{n}\right\}$ and $\left\{\psi_{0}, \psi_{1}, \ldots, \psi_{n}\right\}$ are determined solely by the 
table $A_{n}$ (or $\tilde{A}_{n}$ ) and the values of the functional $M$ on $\mathcal{L}_{n} \cdot \mathcal{L}_{n}$.

Proposition 2.1. The polynomial $P_{n}$ interpolates $\Omega_{M}$ at the table $A_{n}$ in the sense that

$$
\Omega_{M}(z)-P_{n}(z)=z \omega_{n}(z) F_{n}(z)
$$

and the polynomial $Q_{n}$ interpolates $\Omega_{M}$ at the table $\tilde{A}_{n}$ in the sense that

$$
\Omega_{M}(z)-Q_{n}(z)=\frac{\omega_{n}(z)}{z^{n+1}} G_{n}(z) .
$$

Here $F_{n}$ and $G_{n}$ are functions which are defined at least for $\left\{\alpha_{0}, \alpha_{1}, \ldots, \alpha_{n}\right\}$ and $\left\{\alpha_{\infty}, \alpha_{1}, \ldots, \alpha_{n}\right\}$, respectively.

Proof: It can easily be verified by induction that

$$
1+2 \sum_{k=1}^{n} \frac{z \omega_{k-1}(z)}{\omega_{k}(t)}=\frac{t+z}{t-z}\left[1-\frac{z \omega_{n}(z)}{t \omega_{n}(t)}\right]-\frac{z \omega_{n}(z)}{t \omega_{n}(t)}
$$

and

$$
\frac{t+z}{t-z}-\left[1+2 \sum_{k=1}^{n} \frac{z \omega_{k-1}(z)}{\omega_{k}(t)}\right]=\frac{2 z \omega_{n}(z)}{(t-z) \omega_{n}(t)} .
$$

Similarly (e.g. by applying the substar transform) we get

$$
1-2 \sum_{k=1}^{n} \alpha_{k} \frac{t^{k} \omega_{k-1}(z)}{z^{k} \omega_{k}(t)}=-\frac{t+z}{t-z}\left[1-\frac{t^{n+1} \omega_{n}(z)}{z^{n+1} \omega_{n}(t)}\right]-\frac{t^{n+1} \omega_{n}(z)}{z^{n+1} \omega_{n}(t)}
$$

and

$$
\frac{t+z}{t-z}+\left[1-2 \sum_{k=1}^{n} \alpha_{k} \frac{t^{k} \omega_{k-1}(z)}{z^{k} \omega_{k}(t)}\right]=\frac{2 t^{n+1} \omega_{n}(z)}{z^{n}(t-z) \omega_{n}(t)} .
$$

Operating on Equations (2.5) and (2.7) by $M$ we get

$$
\Omega_{M}(z)-\left[\mu_{0}+2 \sum_{k=1}^{n} \mu_{k} z \omega_{k-1}(z)\right]=2 z \omega_{n}(z) M\left[\frac{1}{(t-z) \omega_{n}(t)}\right]
$$

and

$$
\Omega_{M}(z)-\left[\nu_{0}+2 \sum_{k=1}^{n} \nu_{k} \frac{\omega_{k-1}(z)}{z^{k}}\right]=2 \frac{\omega_{n}(z)}{z^{n+1}} M\left[\frac{z t^{n+1}}{(t-z) \omega_{n}(t)}\right]
$$


The left sides of (2.8) and (2.9) are the left sides of (2.2) and (2.3). We introduce

$$
F_{n}(z)=2 M\left[\frac{1}{(t-z) \omega_{n}(t)}\right], G_{n}(z)=2 M\left[\frac{z t^{n+1}}{(t-z) \omega_{n}(t)}\right] .
$$

It follows from the assumption of $M$ being defined on $\tilde{\mathcal{L}} \cdot \tilde{\mathcal{L}}$ (see Section 1) that $F_{n}(z)$ and $G_{n}(z)$ are defined (as finite numbers) for $z=\alpha_{1}, \ldots, \alpha_{n}$. Also both $F_{n}(0)=2 M\left[\frac{1}{t \omega_{n}(t)}\right]$ and $G_{n}(\infty)=2 M\left[\left(\frac{t^{n+1}}{\left(\zeta(t-1) \omega_{n}(t)\right.}\right)_{\zeta=0}\right]$ are defined by assumption.

We shall make use of alternative expressions for $\psi_{n}(z)$ to the one given in the definition (1.11).

Lemma 2.2. Let $m=0,1, \ldots, n-1$. Then we may write

$$
\psi_{n}(z)=M\left[\frac{t+z}{t-z}\left(\frac{\omega_{m}(z)}{\omega_{m}(t)} \varphi_{n}(t)-\varphi_{n}(z) j\right] .\right.
$$

Proof: We may write

$$
\psi_{n}(z)-M\left[\frac{t+z}{t-z}\left(\frac{\omega_{m}(z)}{\omega_{m}(t)} \varphi_{n}(t)-\varphi_{n}(z)\right)\right]=M\left[\frac{(t+z)\left(\omega_{m}(t)-\omega_{m}(z)\right) \varphi_{n}(t)}{(t-z) \omega_{m}(t)}\right] .
$$

The argument of $M$ on the right side is of the form $\frac{R_{m}(t) \varphi_{n}(t)}{\omega_{m}(t)}, R_{m} \in \Pi_{m}$, hence the functional value is zero by orthogonality.

We shall use the following notation:

$$
\begin{aligned}
& \Delta_{n, s}=\psi_{n}(z)+\varphi_{n}(z) P_{s}(z), \\
& \tilde{\Delta}_{n, s}=\psi_{n}(z)+\varphi_{n}(z) Q_{s}(z) .
\end{aligned}
$$

We recall that $p_{n}$ and $q_{n}$ denote the numerator polynomials of $\varphi_{n}$ and $\psi_{n}$, cf. (1.10) and (1.12).

Proposition 2.3. For $m<n$ and arbitrary $s$ the following formula is valid:

$$
q_{n}(z)+p_{n}(z) P_{s}(z)=2 z M\left[\frac{\omega_{s}(t) \omega_{n}(z) \omega_{m}(z) p_{n}(t)-\omega_{s}(z) \omega_{n}(t) \omega_{m}(t) p_{n}(z)}{(t-z) \omega_{s}(t) \omega_{n}(t) \omega_{m}(t)}\right]
$$


Proof: Taking into account (1.20), (1.22) and (2.11) we may write for $m<n$ :

$$
\Delta_{n, s}=M\left[\frac{t+z}{t-z}\left(\frac{\omega_{m}(z)}{\omega_{m}(t)} \varphi_{n}(t)-\varphi_{m i}(z)\right)+\varphi_{n}(z)\left(1+2 \sum_{k=1}^{s} \frac{z \omega_{k-1}(z)}{\omega_{k}(t)}\right)\right]
$$

and by using (2.4) with $n$ replaced by $s$ in the summation we get

$$
\Delta_{n, s}=M\left[\frac{(t+z) \omega_{m}(z) \varphi_{n}(t)}{(t-z) \omega_{m}(t)}-\frac{2 z \omega_{s}(z) \varphi_{n}(z)}{(t-z) \omega_{s}(t)}\right] .
$$

Substitution of the expansion (1.10) for $\varphi_{n}$ then yields

$$
\Delta_{n, s}=M\left[\frac{(t+z) \omega_{s}(t) \omega_{n}(z) \omega_{m}(z) p_{n}(t)-2 z \omega_{s}(z) \omega_{n}(t) \omega_{m}(t) p_{n}(z)}{(t-z) \omega_{s}(t) \omega_{n}(t) \omega_{m}(t) \omega_{n}(z)}\right]
$$

This may again be written as

$$
\begin{aligned}
\Delta_{n, s} & =M\left[\frac{2 z\left[\omega_{s}(t) \omega_{n}(z) \omega_{m}(z) p_{n}(t)-\omega_{s}(z) \omega_{n}(t) \omega_{m}(t) p_{n}(z)\right]}{(t-z) \omega_{s}(t) \omega_{n}(t) \omega_{m}(t) \omega_{n}(z)}\right] \\
& +\omega_{m}(z) M\left[\frac{p_{n}(t)}{\omega_{n}(t) \omega_{m}(t)}\right] .
\end{aligned}
$$

The argument of $M$ in the last term may be written as $\varphi_{n}(t) \frac{1}{\omega_{m}(t)}$, hence the last term is zero since $m<n$. (Note that the argument of the first term to the right in (2.19) belongs to the domain of definition of $M$, since the argument in the last term in (2.19) and the argument in (2.17) (hence also in (2.16) does.) Thus

$$
\Delta_{n, s}=\frac{2 z}{\omega_{n}(z)} M\left[\frac{\omega_{s}(t) \omega_{n}(z) \omega_{m}(z) p_{n}(t)-\omega_{s}(z) \omega_{n}(t) \omega_{m}(t) p_{n}(z)}{(t-z) \omega_{s}(t) \omega_{n}(t) \omega_{m}(t)}\right] .
$$

Multiplication by $\omega_{n}(z)$ gives (2.15).

Proposition 2.4. For $m<n$ and arbitrary $s$ the following formula is valid:

$$
\begin{aligned}
& q_{n}(z)+p_{n}(z) Q_{s}(z) \\
& =\frac{2}{z^{s}} M\left[\frac{t z^{s} \omega_{s}(t) \omega_{n}(z) \omega_{m}(z) p_{n}(t)-t^{s+1} \omega_{s}(z) \omega_{n}(t) \omega_{m}(t) p_{n}(z)}{(t-z) \omega_{s}(t) \omega_{n}(t) \omega_{m}(t)}\right] .
\end{aligned}
$$

Proof: Using (1.21) and (1.23) we get for $m<n$ :

$$
\tilde{\Delta}_{n, s}=M\left[\frac{t+z}{t-z}\left(\frac{\omega_{m}(z)}{\omega_{m}(t)} \varphi_{n}(t)-\varphi_{n}(z)\right)+\left(-1+2 \sum_{k=1}^{s} \alpha_{k} \frac{t^{k} \omega_{k-1}(z)}{z^{k} \omega_{k}(t)}\right)\right]
$$


and by (2.6) with $n$ replaced by $s$ in the summation this yields

$$
\tilde{\Delta}_{n, s}=M\left[\frac{(t+z) \omega_{m}(z) \varphi_{n}(t)}{(t-z) \omega_{m}(t)}-\frac{2 t^{s+1} \omega_{s}(z) \varphi_{n}(z)}{z^{s}(t-z) \omega_{s}(t)}\right]
$$

This may be written as

$$
\tilde{\Delta}_{n, s}=M\left[\frac{(t+z) z^{s} \omega_{s}(t) \omega_{n}(z) \omega_{m}(z) p_{n}(t)-2 t^{s+1} \omega_{s}(z) \omega_{n}(t) \omega_{m}(t) p_{n}(z)}{z^{s}(t-z) \omega_{s}(t) \omega_{n}(t) \omega_{m}(t) \omega_{n}(z)}\right] .
$$

Rearranging we get

$$
\begin{aligned}
& \tilde{\Delta}_{n, s}=M\left[\frac{2\left[t z^{s} \omega_{s}(t) \omega_{n}(z) \omega_{m}(z) p_{n}(t)-t^{s+1} \omega_{s}(t) \omega_{n}(t) \omega_{m}(t) p_{n}(z)\right]}{z^{s}(t-z) \omega_{s}(t) \omega_{n}(t) \omega_{m}(t) \omega_{n}(z)}\right] \\
& -\omega_{m}(z) M\left[\frac{p_{n}(t)}{\omega_{n}(t) \omega_{m}(t)}\right] .
\end{aligned}
$$

The last term is zero (cf. the proof of Proposition 2.3), and so (2.4) follows by multiplication by $\omega_{n}(z)$.

For each $n$ we define interpolation tables $B_{2 n-1}^{(n)}$ and $\tilde{B}_{2 n-1}^{(n)}$ by

$$
\begin{aligned}
& B_{2 n-1}^{(n)}=\left\{\alpha_{0}, \beta_{1}^{(n)}, \beta_{2}^{(n)}, \ldots, \beta_{2 n-1}^{(n)}\right\} \\
& \tilde{B}_{2 n-1}^{(n)}=\left\{\alpha_{\infty}, \beta_{1}^{(n)}, \beta_{2}^{(n)}, \ldots, \beta_{2 n-1}^{(n)}\right\}
\end{aligned}
$$

where

$$
\beta_{k}^{(n)}=\alpha_{k} \text { for } \emptyset \leq k \leq n, \beta_{k}^{(n)}=\alpha_{k-n} \text { for } n<k \leq 2 n-1 .
$$

We further define

$$
\omega_{0}^{(n)}=1, \omega_{k}^{(n)}(z)=\prod_{j=1}^{k}\left(z-\beta_{j}^{(n)}\right) \text { for } k=1,2, \ldots, 2 n-1 .
$$

Note that

$$
\omega_{k}^{(n)}(z)=\omega_{n}(z) \omega_{k-n}(z) \text { for } n<k \leq 2 n-1
$$


We also define moments $\mu_{k}^{(n)}, \nu_{k}^{(n)}$ by

$$
\begin{gathered}
\mu_{k}^{(n)}=M\left[\frac{1}{\omega_{k}^{(n)}(t)}\right], k=0,1,2, \ldots, 2 n-1, \\
\nu_{0}^{(n)}=-\mu_{0}^{(n)}, \quad \nu_{k}^{(n)}=M\left[\frac{\alpha_{k} t^{k}}{\omega_{k}^{(n)}(t)}\right], k=1,2, \ldots, 2 n-1 .
\end{gathered}
$$

Then $\mu_{k}^{(n)}=\mu_{k}, \nu_{k}^{(n)}=\nu_{k}$ for $k=0,1, \ldots, n$. Finally we introduce polynomials $P_{2 n-1}^{(n)}$ and $Q_{2 n-1}^{(n)}$ defined by

$$
\begin{aligned}
& P_{2 n-1}^{(n)}(z)=\mu_{0}^{(n)}+2 \sum_{k=\emptyset_{1}}^{2 n-1} \mu_{k}^{(n)} z \omega_{k-1}^{(n)}(z), \\
& Q_{2 n-1}^{(n)}(z)=\nu_{0}^{(n)}+2 \sum_{k=\oint_{1}}^{2 n-1} \nu_{k}^{(n)} \frac{\omega_{k-1}^{(n)}(z)}{z^{k}} .
\end{aligned}
$$

Proposition 2.5. The polynomial $P_{2 n-1}^{(n)}(z)$ interpolates $\Omega_{M}(z)$ in the sense that

$$
\Omega_{M}(z)-P_{2 n-1}^{(n)}(z)=z \omega_{n}(z) \omega_{n-1}(z) f_{2 n-1}(z)
$$

and the polynomial $Q_{2 n-1}^{(n)}(z)$ interpolates $\Omega_{M}(z)$ in the sense that

$$
\Omega_{M}(z)-Q_{2 n-1}^{(n)}(z)=\frac{\omega_{n}(z) \omega_{n-1}(z)}{z^{2 n}} g_{2 n-1}(z) .
$$

Here $f_{2 n-1}(z)$ has finite values for $z=\alpha_{0}, \alpha_{1}, \ldots, \alpha_{n}$, and $g_{2 n-1}(z)$ has finite values for $z=\alpha_{\infty}, \alpha_{1}, \ldots, \alpha_{n}$.

Proof: This follows by applying Proposition 2.1 to the tables $B_{2 n-1}^{(n)}$ and $\tilde{B}_{2 n-1}^{(n)}$, taking (2.30) into account.

Proposition 2.6. The following formula is valid:

$$
q_{n}(z)+p_{n}(z) P_{2 n-1}^{(n)}(z)=z \omega_{n}(z) \omega_{n-1}(z) F_{2 n-1}(z),
$$

where $F_{2 n-1}(z)$ has finite values for $z=\alpha_{0}, \alpha_{1}, \ldots, \alpha_{n}$. 
Proof: It follows by applying Proposition 2.3 to the table $B_{2 n-1}^{(n)}$, setting $m=n-1, s=$ $2 n-1$ and taking into account (2.30), that

$$
q_{n}(z)+p_{n}(z) P_{2 n-1}^{(n)}(z)=2 z \omega_{n}(z) \omega_{n-1}(z) M\left[\frac{p_{n}(t)-p_{n}(z)}{(t-z) \omega_{n}(t) \omega_{n-1}(t)}\right] .
$$

The function $F_{2 n-1}(z)=2 M\left[\frac{p_{n}(t)-p_{n}(z)}{(t-z) \omega_{n}(t) \omega_{n-1}(t)}\right]$ is defined and finite at $z=\alpha_{0}, \alpha_{1}, \ldots, \alpha_{n}$ by assumption, the expression in brackets belonging to the domain of definition of $M$.

Proposition 2.7. The following formula is valid:

$$
q_{n}(z)+p_{n}(z) Q_{2 n-1}^{(n)}(z)=\frac{\omega_{n}(z)}{z} G_{2 n-1}(z),
$$

where $G_{2 n-1}(z)$ has finite values for $z=\alpha_{\infty}, \alpha_{1}, \ldots, \alpha_{n}$.

Proof: It follows by applying Proposition 2.4 to the table $\tilde{B}_{2 n-1}^{(n)}$, setting $m=0, s=$ $2 n-1$ and taking into account $(2.30)$, that

$$
q_{n}(z)+p_{n}(z) Q_{2 n-1}^{(n)}=2 \frac{\omega_{n}(z)}{z} M\left[\frac{t z^{2 n-1} \omega_{n-1}(t) p_{n}(t)-t^{2 n} \omega_{n-1}(z) p_{n}(z)}{(t-z) \omega_{n}(t) \omega_{n-1}(t) z^{2 n-2}}\right] .
$$

The function $G_{2 n-1}(z)=2 M\left[\frac{t z^{2 n-1} \omega_{n-1}(t) p_{n}(t)-t^{n} \omega_{n-1}(z) p_{n}(z)}{(t-z) \omega_{n}(t) \omega_{n-1}(t) z^{2 n-2}}\right]$ is defined and finite for $z=\alpha_{1}, \ldots, \alpha_{n}$. Furthermore

$H_{2 n-1}(\zeta)=G_{2 n-1}\left(\zeta^{-1}\right)=2 M\left[\frac{t \omega_{n-1}(t) p_{n}(t)-\zeta^{2 n-1} t^{2 n} \omega_{n-1}\left(\zeta^{-1}\right) p_{n}\left(\zeta^{-1}\right)}{(t \zeta-1) \omega_{n}(t) \omega_{n-1}(t)}\right]$ is finite for $\zeta=$ 0 , since argument for $M$ in this expression belong to the domain of definition of $M$ for $\zeta=0$.

Theorem 2.8. The rational function $\frac{q_{n}(z)}{p_{n}(z)}=\frac{\psi_{n}(z)}{\varphi_{n}(z)}$ interpolates $-\Omega_{M}(z)$ linearly at the table $\left\{\alpha_{0}, \alpha_{\infty}, \alpha_{1}, \alpha_{1}, \alpha_{2}, \alpha_{2}, \ldots, \alpha_{n-1}, \alpha_{n-1}, \alpha_{n}\right\}$ in the sense that

$$
q_{n}(z)+p_{n}(z) \Omega_{M}(z)=A_{n}(z) z \omega_{n}(z) \omega_{n-1}(z)
$$

and

$$
q_{n}(z)+p_{n}(z) \Omega_{M}(z)=B_{n}(z) \cdot \frac{\omega_{n}(z)}{z}
$$


where $A_{n}(z)$ and $B_{n}(z)$ are functions such that $A_{n}(z)$ is finite for $z=\alpha_{0}, \alpha_{1}, \ldots, \alpha_{n}$ and $B_{n}(z)$ is finite for $z=\alpha_{\infty}, \alpha_{1}, \ldots, \alpha_{n}$.

Proof: We may write

$$
q_{n}(z)+p_{n}(z) \Omega_{M}(z)=q_{n}(z)+p_{n}(z) P_{2 n-1}^{(n)}(z)+p_{n}(z)\left[\Omega_{M}(z)-P_{2 n-1}^{(n)}(z)\right] .
$$

Formula (2.41) now follows from (2.35) and (2.37). Similarly we may write

$$
q_{n}(z)+p_{n}(z) \Omega_{M}(z)=q_{n}(z)+p_{n}(z) Q_{2 n-1}^{(n)}(z)+p_{n}(z)\left[\Omega_{M}(z)-Q_{2 n-1}^{(n)}(z)\right]
$$

and so Formula (2.42) follows from (2.36) and (2.39).

Remark. The rational function $\frac{p_{n}(z)}{q_{n}(z)}$ satisfies $2 n+1$ interpolation conditions for $-\Omega_{M}(z)$, while the degrees of the polynomials $p_{n}(z)$ and $q_{n}(z)$ are both at most $n$. Thus $\frac{p_{n}(z)}{q_{n}(z)}$ is a multipoint Padé approximant to $-\Omega_{M}(z)$.

Instead of introducing the new tables $B_{2 n-1}^{(n)}$ and $\tilde{B}_{2 n-1}^{(n)}$ for each $n$ we might introduce one new table $B=\left\{\alpha_{0}, \alpha_{\infty}, \alpha_{1}, \alpha_{1}, \alpha_{2}, \alpha_{2}, \ldots, \alpha_{n}, \alpha_{n}, \ldots\right\}$. Orthogonality would be defined in terms of the table $A=\left\{\alpha_{0}, \alpha_{1}, \alpha_{2}, \ldots, \alpha_{n}, \ldots\right\}$, interpolation would be at points of $B$. Moments, however, would have to be defined in terms of the tables $B_{2 n-1}^{(n)}, \tilde{B}_{2 n-1}^{(n)}$.

\section{REFERENCES}

1. N.I. Akhiezer, The classical moment problem and some related questions in analysis, Hafner, New York, 1965.

2. C. Brezinski, Padé-Type Approximants and Orthogonal Polynomials, Birkhäuser, Basel/Boston/Stuttgart, 1980.

3. A. Bultheel, Laurent series and their Padé approximations, Operator Theory: Advances and Applications, vol. 27, Birkhäuser Verlag, Basel, 1987.

4. A. Bultheel and P. Dewilde, Orthogonal functions related to the Nevanlinna-Pick problem, Mathematical Theory of Networks and Systems, Proceedings MTNS Con- 
ference, Delft, The Netherlands (Ed. P. Dewilde) Western Periodicals, North Hollywood, 1979, 207-211.

5. A. Bultheel, P. Gonzalez-Vera, E. Hendriksen and O. Njåstad, A Szegö theory for rational functions, Technical Report TW-131, K.U. Leuven, Dept. of Computer Science, May 1990.

6. A. Bultheel, P. Gonzalez-Vera, E. Hendriksen and O. Njåstad, The computation of orthogonal rational functions and their interpolating properties, Numerical Algorithms 2 (1992) 85-114.

7. A. Bultheel, P. Gonzalez-Vera, E. Hendriksen and O. Njåstad, Moment problems and orthogonal functions, J. Comp. Appl. Math., to appear.

8. A. Bultheel, P. Gonzalez-Vera, E. Hendriksen and O. Njåstad, Orthogonal rational functions with poles on the unit circle, J. Math. Anal. Appl., to appear.

9. A. Bultheel, P. Gonzalez-Vera, E. Hendriksen and O. Njåstad, A uniform approach to orthogonal rational functions on the unit circle and the unit line, to appear.

10. T.S. Chihara, Introduction to Orthogonal Polynomials, Mathematics and Its Applications Series, Gordon \& Breach, New York/London/Paris, 1978.

11. M.M Djrbashian, A survey on the theory of orthogonal systems and some open problems, Orthogonal Polynomials: Theory and Practice (Ed. P. Nevai, Kluwer, Dordrecht/Boston/London 1990) 135-146.

12. M.A. Gallucci and W.B. Jones, Rational Approximations corresponding to Newton series (Newton-Padé approximants), J. Approximation Theory 17 (1976) 366-392.

13. W.B. Jones, O. Njåstad and W.J. Thron, Orthogonal Laurent polynomials and the strong Hamburger moment problem, J. Math. Anal. Appl. 98 (1984) 528-554.

14. O. Njåstad, An extended Hamburger moment problem, Proc. Edinburgh Math. Soc. Ser. II, 28 (1985) 167-183. 
15. O. Njåstad, Multipoint Padé approximation and orthogonal rational functions, Non -linear Numerical Methods and Rational Approximation (ed.: A. Cuyt, D. Reidel Publ. Comp., Dordrecht 1988) 259-270.

16. O. Njåstad, A modified Schur algorithm and an extended Hamburger moment problem, Trans. Amer. Math. Soc. 327 (1991) 283-311.

17. O. Njåstad and W.J. Thron, The theory of sequences of orthogonal L-polynomials, in Padé Approximants and Continued Fractions (H. Waadeland and H. Walling, Det Kongelige Norske Videnskabers Selskab, Skrifter, 1983). 